\title{
Analysis of Modified Passive Safety System in Fast Rectors Transients
}

\author{
Carlo Oggioni $^{1}$, Chris Keckler ${ }^{2}$, and Massimiliano Fratoni ${ }^{2}$ \\ ${ }^{1}$ Politecnico di Milano \\ Building B12, 34 La Masa street, Milano, 20156 Italy \\ ${ }^{2}$ University of California, Berkeley \\ Etcheverry Hall, 2521 Hearst Ave, Berkeley, CA 94709, USA
}

carlo.oggioni@berkeley.edu, keckler@berkeley.edu, maxfratoni@berkeley.edu,

\begin{abstract}
The Autonomous Reactivity Control (ARC) system is a passive safety system aiming to provide an additional negative reactivity feedback during reactor transient scenarios. This paper shows how the performance of the ARC system can be enhanced by introducing a hydraulic diode that allows for different engagement and disengagement speeds of system. The benefits of the proposed system is assessed in a reference soidum-cooled fast reactor (SFR) during multiple postulated transient scenarios. The reactor and plant dynamic response are evaluated using SAS4A/SASSYS-1, whereas for the internal ARC system fluid dynamics, the SAM code is adopted. The two codes are externally coupled using a custom driver script which coordinates data exchange and restart calculations at each time step, with Picard iterations used to converge each time step. All the transients analyzed in this work show that the enhanced ARC system is effective in reducing peak temperatures and in reducing the oscillatory behavior encountered in some cases with the standard ARC system.
\end{abstract}

KEYWORDS: Passive safety, ARC system, SAS4A/SASSYS-1, SAM, Coupled calculation, Sodium-cooled Fast Reactor

\section{INTRODUCTION}

Although other methods have been proposed for achieving passive safety, many of them involve an increase of neutron leakage or an addition of moderating material which are generally undesirable because many of the benefits of the fast spectrum are impeded. Other proposed methods require adding a new engineered system to compensate the coolant void feedback (e.g., control rods actuated by the Curie point tested in JOYO [1] or the Gas Expansion Modules (GEMs) tested in FFTF [2]). These systems have been proven effective, nevertheless, they suffer from certain limitations. The Curie point system actuation is irreversible and GEMs are activated only by a reduction of flow. One engineered passive safety system that overcomes the major issues is an evolution of the Lithium Expansion Module (LEM) [3], referred to as the Autonomous Reactivity Control system. The ARC system, developed by Qvist et al. [4], is autonomously actuated by a coolant temperature increase. This temperature increase passively induces thermal expansion of the ARC system's working fluid that results in the insertion of the absorbing liquid into tubes penetrating through the 


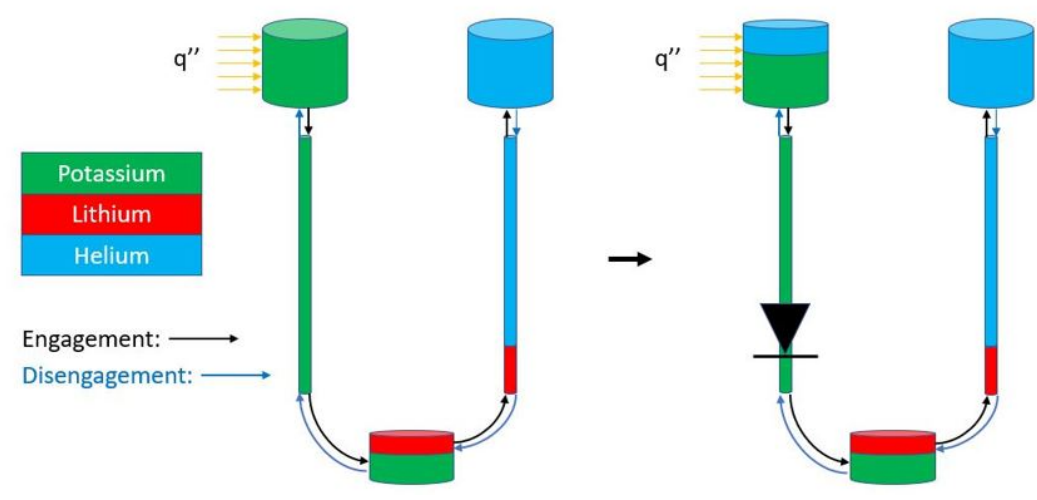

Figure 1: Abstraction of the ARC system (left) and its modification to include the one-way valve and cover gas (right).

active core. Previous work [5] has demonstrated that larger margins to boiling and melting during various transients in SFR can be gained through the inclusion of an ARC system. Nevertheless, challenges related to the potential of inducing oscillatory behavior have been observed under certain situations. This study proposes the addition of an hydraulic diode to the standard ARC system, and shows, using coupled simulations of various unprotected transients, that such enhanced design can reduce, if not eliminate, the oscillatory behavior described above.

\section{PRESENTATION OF THE ARC SYSTEM}

The ARC system is a design evolution of a standard fuel assembly and is composed of two parts: an upper section located at the top of the assembly and composed of two reservoirs, one filled with liquid and the other with inert gas; a lower part consisting of a single reservoir located at the bottom of the fuel assembly and filled with liquid. The two parts are linked with two concentric tubes. The reservoirs are filled with three fluids: an expansion liquid (potassium), present in the upper part, in the liquid reservoir, and in the inner tube; an absorber liquid, (lithium), present in the lower reservoir on top of the expansion liquid; an inert gas (helium), present in the outer tube and the gas reservoir in the upper part. In Figure 1, an abstracted diagram of the ARC system is shown. In the Figure, the two concentric tubes are separated and the upper reservoir is shown divided into its two portions.

An ideal ARC system behaves as follows. When during an abnormal condition the temperature in the core rises and consequently the coolant heats up, the coolant exchanges heat to the upper reservoir and the expansion liquid, which thermally expands and is forced into the lower reservoir pushing the absorber liquid into the active core while compressing the inert gas above. The absorber liquid absorbs neutrons in the core, introducing a negative reactivity that arrests the transient and brings the core to a stable critical configuration. As the core cools down, the temperature of the expansion liquid starts to fall, allowing the level of the absorber liquid to decrease. More details about the ARC system can be found in [5].

Previous work [5] has shown the effectiveness of the ARC system in gaining margins to coolant 
boiling and fuel melting for different postulated accident scenarios. However, it has also underlined an oscillatory behavior that may lead to coolant boiling under certain circumstances. The reason for this behavior is the strong coupling of the ARC system to fluctuations in core temperature. The ARC system is designed to be strongly coupled to an increase in temperature to provide negative reactivity during transients. However once the core decreases its temperature, due to such strong coupling, the ARC system will be disengaged, reducing the negative reactivity and possibly allowing for another increase in core temperature. Under certain conditions, this process repeats and may lead to diverging oscillatory behavior; therefore, it is desired to remove these oscillations by differentiating the engagement and the disengagement of the ARC.

To achieve this aim, the introduction of a one-way valve in the inner tube is proposed, allowing unimpeded flow in one direction but constricted flow in the other, similar to a hydraulic diode. This way the insertion of the neutronic poison is still quick but the withdrawal is hindered, leaving the lithium for more time in the core region and potentially eliminating oscillations. To ensure proper behavior of the one-way valve, a cover gas is added above potassium in the upper reservoir. This cover gas is needed because with the upper reservoir filled only with liquid, the actuation speed depends only on the heat exchange rate and the presence of the valve does not influence the flow. By adding the cover gas, the one-way valve is able to influence the actuation speed of the ARC system. Figure 1 presents an abstract diagram of the ARC system with the modifications needed to include the one-way valve and the second cover gas.

In the ARC system a number of parameters can be modified to change the actuation characteristics. In this analysis, the different design choices are lumped into three parameters: (1) total system worth (w [\$]), (2) volume of the cover gas in the upper reservoir originally filled with liquid (cover-volume $\left.\left[\mathrm{m}^{3}\right]\right)$, and (3) loss coefficients for the engagement and the disengagement $\left(\mathrm{K}_{\text {enga }}\right.$, $\mathrm{K}_{\text {dise }}$ ). The total system worth is varied by modifying the volume of absorber liquid in the core during full actuation and the enrichment level of the absorber liquid. The volume of the cover gas largely influences the speed of actuation, which to a smaller extent is influenced by other parameters such as the diameter of the tubes and the heat transfer coefficient from the coolant to the upper reservoir. This study ignores those other parameters and alters the actuation speed only via the cover gas volume, assuming a fixed heat-transfer coefficient between the coolant and the upper reservoir which has been determined previously using Computational Fluid Dynamics simulations.

Additional parameters that primarily characterize the enhanced ARC system are the loss coefficients, which depend largely on the one-way valve design. Since the ARC system is intended to be a passive system, the one-way valve should not have any active components. Additionally, its design should not include mechanical components that could easily fail, and it should be able to achieve the desired loss coefficients. A possible choice is shown in Figure 2 in a simplified form. In the figure, the line on the right, at the exit of the valve, represents a grate that holds the poppet inside the valve during engagement. This valve concept was used to evaluate the loss coefficients implemented in the numerical calculations of this study. To evaluate the $\mathrm{K}_{\text {dise }}$, an orifice with sudden change in velocity and in flow area was used as reference. In particular, a thick edged orifice in a straight tube was chosen, as in Figure 3, and the loss coefficients were evaluated according to [8].

Finally, one more design parameter is the distance between the top of the absorber fluid and the 


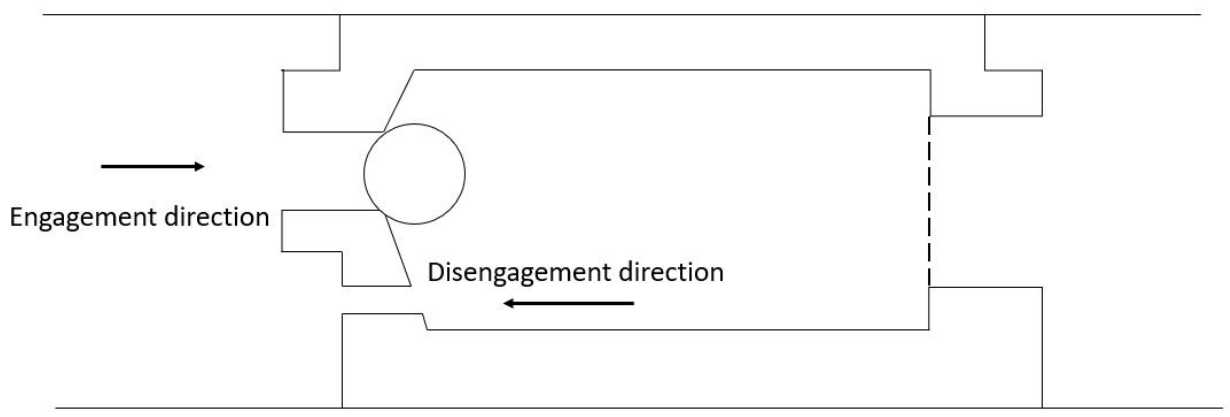

Figure 2: Possible one-way valve to be used inside the ARC system.

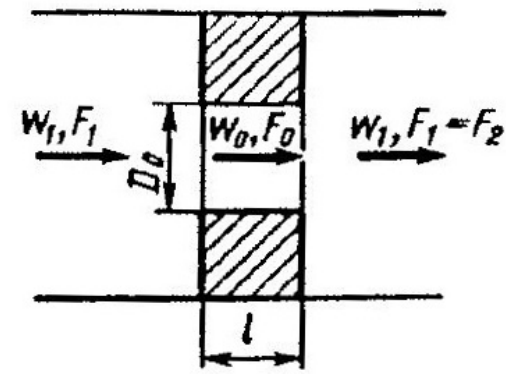

Figure 3: Orifice in a straight tube to evaluated the $\mathbf{K}_{d i s e}[8]$.

bottom of the active core. This was ignored in this study, as it was previously shown to have a small impact on the ARC system performance.

\section{METHODOLOGY AND SOFTWARE DESIGN}

The performance of the modified ARC system is tested by simulating different postulated accident scenarios in a SFR. To evaluate the reactor and plant dynamic response the SAS4A/SASSYS-1 [6] (called SAS for simplicity) code is used, whereas for the internal ARC system fluid dynamics, the SAM code [7] is adopted. Although developed for single-phase incompressible flow, SAM offers the ability to model liquid volumes with cover gas on top, tracking the liquid level in the volumes using a simplified algorithm. This type of component is used to model the upper reservoir and the outer concentric tube, allowing for the level of the absorber fluid to be obtained during the transients. This level is then converted to a reactivity insertion value using a table of worth versus absorber insertion level.

Regarding the one-way valve, in SAM it is possible to model a minor flow loss as $\Delta P=K \rho u^{2} / 2$, with different values of $\mathrm{K}$ for the engagement and the disengagement. For the disengagement, the orifice model shown in Figure 3 is used. Although the evaluation of $\mathrm{K}_{\text {enga }}$ is more complicated due to the presence of a grate and the poppet, the pressure loss should be much smaller due to the large flow area through the valve in the forward-flow direction; therefore, a difference of 3- 


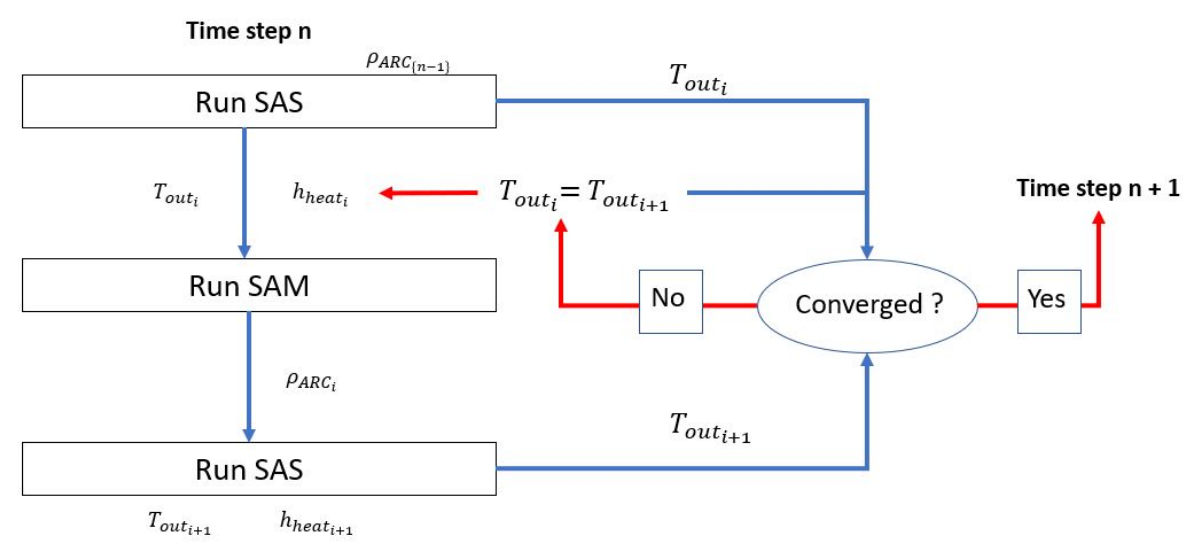

Figure 4: Picard iteration algorithm used in the external coupling.

4 orders of magnitude between $\mathrm{K}_{\text {dise }}$ and $\mathrm{K}_{\text {enga }}$ is assumed for this study. The two codes are externally coupled using a custom driver script which exchange data and restart calculations at each time step. From the SAS calculation, the core outlet temperature and flow rate values are obtained and used to establish boundary conditions as input to SAM. From SAM, the absorber liquid level is obtained and converted offline to a reactivity insertion, which is used as SAS input. Picard iterations are used to converge each time step, as shown in Figure 4. In each time step SAS runs at least two times. The convergence is checked on the value $T_{\text {out }}$ calculated by SAS. When err $=\left(\left|T_{\text {out }_{i}}-T_{\text {out }_{i+1}}\right|\right) / T_{\text {out }} \geq 10^{-4}=$ err $_{\text {limit }}, T_{\text {out }_{i+1}}$ is used as new input to SAM and the convergence is checked again using $T_{\text {out }}$ i+1 $_{1}$ and $T_{\text {out }}{ }_{i+2}$. The external coupling is used for this study because it does not require access to or modifications of the source code, however it is a slow process because of the re-initialization of the codes in each time step, particularly for SAM.

\section{REFERENCE REACTORS AND TRANSIENTS}

The performance of the enhanced ARC system is evaluated a medium-sized oxide-fueled Advanced Burner Reactor (ABR) designed by Argonne National Laboratory (details about the core can be found in [4]). Three different transients are examined: Unprotected Loss of Heat Sink (ULOHS), Unprotected Loss of Flow (ULOF), and Unprotected Transient Overpower (UTOP). Here the term unprotected means that the reactor system fails to scram. A different set of assumptions is used for each transient, as provided in Table 1. These accidents were chosen because they test the ARC system behavior on a wide range of different situations concerning reactivity introduction, coolant temperature increases, and loss of flow. Moreover, these transients have a long history of simulation and validation with SAS, ensuring the applicability of the computational tools employed in this study. The validation was conducted with data from the Experimental Breeder Reactor II (EBR-II) tests conducted in the 1980s [9].

\section{RESULTS}

A summary of the transient results are shown in Table 2. Without the ARC system, coolant boiling does not occur in any of the three unprotected transients. The smallest margin to coolant boiling 
Table 1: Assumptions specific to each of the three transients examined.

\begin{tabular}{llll}
\hline & UTOP & ULOF & ULOHS \\
\hline Primary Loop Heat Rejection & Nominal & Nominal & Zero \\
Pump Speed & Nominal & 20s halving time & Nominal \\
Control System & $\$ 0.75$ inserted at $0.005 \$ / \mathrm{s}$ & No scram & No scram \\
\hline
\end{tabular}

was obtained in the ULOF accident, whereas the smallest margin to fuel melting was found in the UTOP accident. The introduction of the ARC system in the ABR core allows for a significant safety margin to be gained, whereas with the standard configuration, an oscillatory behavior was observed in the ULOF and UTOP transients, as demonstrated in Figure 5. In this Figure $w$ is set to $\$ 0.87$, which was found to be the maximum value of $w$ before the oscillations became strong enough to induce boiling. A parametric study was conducted in previous studies to analyze the transients varying the former ARC parameters, and it was observed that at high $w$ these strong oscillations were prone to occur in both the ULOF and UTOP accidents. As seen from Table 2, the modification of the ARC system leads to higher margins. The enhanced ARC system, indeed, reduces the oscillation behavior and $w$ can be increased beyond the previously obtainable values. As $w$ depends on a number of physical design parameters, the detailed calculations needed to determine an upper bound on its value are outside the scope of this study. The maximum value for this study was assumed equal to $\$ 1.5$. A parametric study was performed for the enhanced ARC system (Figure 6) to evaluate the configuration with the highest margin and to ensure that the oscillations do not diverge with different configurations. The value of $\mathrm{K}_{\text {enga }}=10^{4}$ and $\mathrm{K}_{\text {dise }}=10^{8}$ were kept constants, as they were shown to sufficiently reduce oscillatory behavior. The value of $\mathrm{K}_{\text {dise }}$ was determined assuming a hole of $0.1 \mathrm{~mm}$. $w$ was varied from $\$ 1$ to $\$ 1.5$ and the cover volume from $5 \times 10^{-5}$ to $4 \times 10^{-4} \mathrm{~m}^{3}$. The yardstick for this parametric study was the peak coolant temperature in the ULOF transient whose margin, as shown in Table 2, is the smallest. For the enhanced ARC system, the highest margins were found with the smaller cover gas $\left(5 \times 10^{-5} \mathrm{~m}^{3}\right)$ and high reactivity $(\$ 1.5)$, which allows for a system that is activated both quickly and strongly. As seen in Figure 6, no unstable behavior was observed over the design space examined. Furthermore, in this study, very little sensitivity to the total system worth was observed with big cover volumes.

\section{CONCLUSIONS AND FUTURE WORK}

This work proposes the use of a hydraulic diode valve in the ARC system so to mitigate the oscillations observed in previous studies of ULOF and UTOP transients in SFRs. The analysis of such enhanced ARC system shows that the introduction of a one-way valve reduces the oscillatory behavior and completely prevent the diverging oscillations that lead to coolant boiling. In the reference $\mathrm{ABR}$ core used for testing, the enhanced ARC system has enabled a more aggressive ARC system design compared to what was previously achievable, leading to significantly higher margins to both coolant boiling and fuel melting. Boiling was not observed for all of the configurations tested and parametric studies showed that the highest margins are found at the edge of the study domain; therefore, future works could extend the design domain to possibly achieve higher margins and to be sure that the oscillations do not diverge with even more aggressive designs. 
Table 2: Peak temperatures in the in ABR core.

\begin{tabular}{lllll}
\hline & $\begin{array}{l}\text { Peak coolant } \\
\text { temp }\left[{ }^{\circ} \mathrm{C}\right]\end{array}$ & $\begin{array}{l}\text { Peak fuel } \\
\text { temp }\left[{ }^{\circ} \mathrm{C}\right]\end{array}$ & $\begin{array}{l}\text { Margin to } \\
\text { boiling }\left[{ }^{\circ} \mathrm{C}\right]\end{array}$ & $\begin{array}{l}\text { Margin to } \\
\text { fuel melting }\left[{ }^{\circ} \mathrm{C}\right]\end{array}$ \\
\hline \multicolumn{6}{c}{ Peak temperatures in ABR core without the ARC system } \\
\hline ULOHS & 722 & 1724 & 311 & 1026 \\
UTOP & 623 & 2089 & 389 & 661 \\
ULOF & 813 & 1724 & 144 & 1026 \\
\hline \multicolumn{6}{c}{ Peak coolant } & Peak fuel & Margin to & Margin to \\
& temp $\left[{ }^{\circ} \mathrm{C}\right]$ & temp $\left[{ }^{\circ} \mathrm{C}\right]$ & boiling gained $\left[{ }^{\circ} \mathrm{C}\right]$ & melting gained $\left[{ }^{\circ} \mathrm{C}\right]$ \\
\hline \multicolumn{6}{c}{ Peak temperatures in ABR core with the standard ARC system } \\
\hline ULOHS & 635 & 1724 & 73 & - \\
UTOP & 590 & 1900 & 31 & 189 \\
ULOF & 705 & 1724 & 103 & - \\
\hline \multicolumn{6}{c}{ Peak temperatures in ABR core with the enhanced ARC system } \\
\hline ULOHS & 561 & 1724 & 143 & - \\
UTOP & 566 & 1729 & 51 & 360 \\
ULOF & 621 & 1724 & 183 & - \\
\hline
\end{tabular}

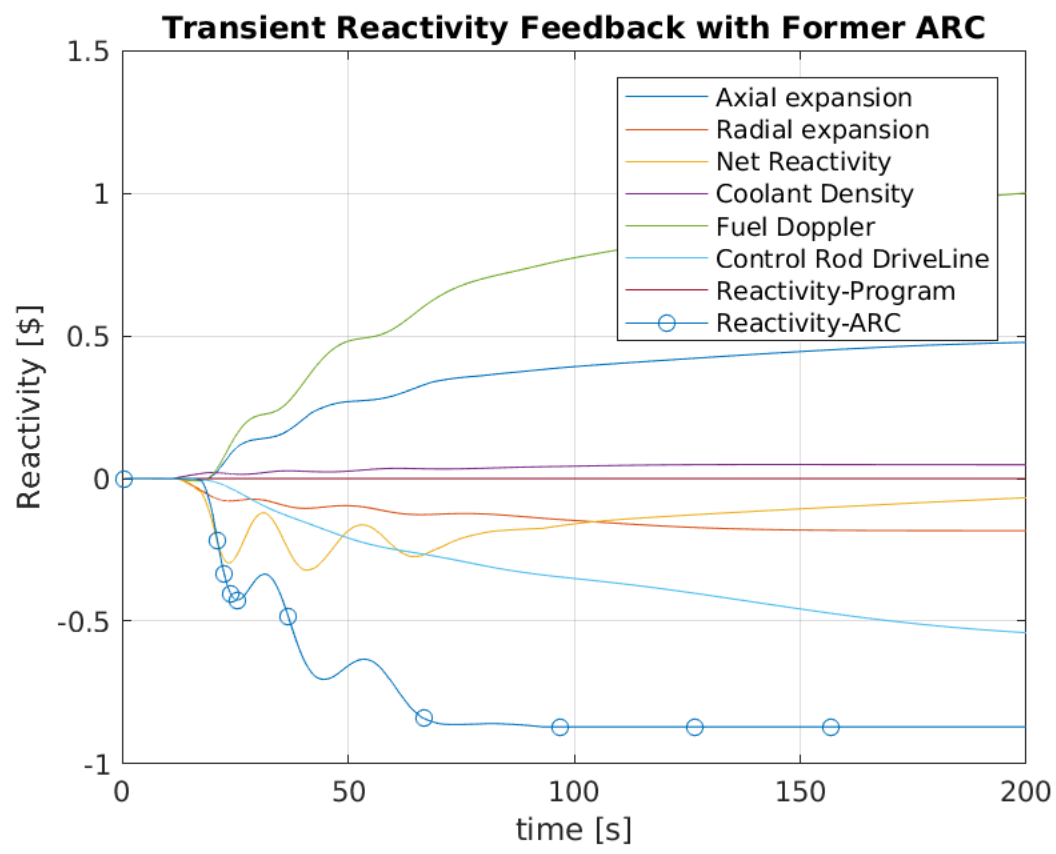

Figure 5: Response to a ULOF accident in ABR core with the standard ARC system. 


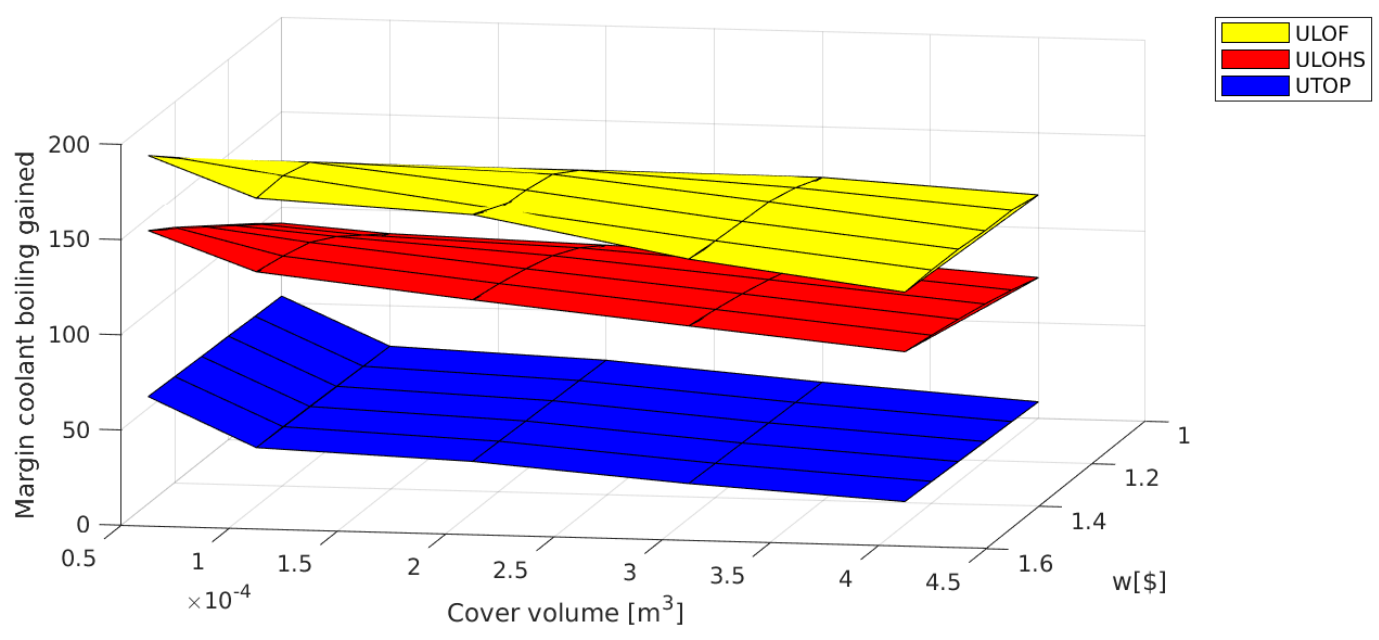

Figure 6: Coolant margin in ABR core with the enhanced ARC system.

Additionally, more detailed studies should be performed on the design of the one-way valve to find such a design with the desired loss coefficients that satisfies all operational, manufacturing, and engineering constraints. Finally, work to validate the computational scheme employed in this analysis is of high importance, although the general conclusions of this study are anticipated to be valid regardless.

\section{REFERENCES}

[1] M. Takamatu, T. Sekine, T. Aoyama, and M. Uchida, "Demonstration of Control Rod Holding Stability of the Self Actuated Shutdown System in Joyo for Enhancement of Fast Reactor Inherent Safety" Journal of Nuclear Science and Technology, 44, 3, 511-517 (2007).

[2] A. E. Waltar, D. R. Todd, P. V. Tsvetkov, Fast spectrum reactors, New York (2012).

[3] M. Kambe and M. Uotani, "Design and Development of Fast Breeder Reactor Passive Reactivity Control Systems: LEM and LIM," Nuclear Technology, 122, 2, 179-195 (May 1998).

[4] S. Qvist and E. Greenspan, "An Autonomous Reactivity Control system for improved fast reactor safety," Progress in Nuclear Energy, 77 (2014).

[5] E. Greenspan, T. Fanning, M. Fratoni, M. Gradecka, C. Keckler, S. Qvist Enhanced Performance Fast Reactors with Engineered Passive Safety System, Final report, University of California, Berkeley, (December 2018).

[6] J. Cahalan and T. H. Fanning, The SAS4A / SASSYS-1 Safety Analysis Code System, Tech. rep., Argonne National Laboratory, Lemont, IL (2012).

[7] Rui Hu, SAM Theory Manual, Tech. rep., Argonne National Laboratory, Lemont, IL (2017).

[8] I. E. Idelchik, Handbook of Hydraulic Resistance 3rd edition, (1994).

[9] P. Herzog, L. K. Chang, E. M. Dean, E. E. Feldman, D. J. Hill, D. Mohr, H. P. Planchon, CODE VALIDATION WITH EBR.II TEST DATA, Argonne National Laboratory, Lemont, IL (1992). 